\title{
The Existence, Uniqueness, and Controllability of Neutral Stochastic Delay Partial Differential Equations Driven by Standard Brownian Motion and Fractional Brownian Motion
}

\author{
Dehao Ruan and Jiaowan Luo \\ Department of Probability and Statistics, School of Mathematics and Information Sciences, Guangzhou University, \\ Guangzhou, Guangdong 510006, China \\ Correspondence should be addressed to Jiaowan Luo; jluo@gzhu.edu.cn
}

Received 30 November 2017; Revised 5 January 2018; Accepted 7 February 2018; Published 2 April 2018

Academic Editor: Chris Goodrich

Copyright (C) 2018 Dehao Ruan and Jiaowan Luo. This is an open access article distributed under the Creative Commons Attribution License, which permits unrestricted use, distribution, and reproduction in any medium, provided the original work is properly cited.

\begin{abstract}
We focus on a class of neutral stochastic delay partial differential equations perturbed by a standard Brownian motion and a fractional Brownian motion. Under some suitable assumptions, the existence, uniqueness, and controllability results for these equations are investigated by means of the Banach fixed point method. Moreover, an example is presented to illustrate our main results.
\end{abstract}

\section{Introduction}

Fractional Brownian motion (fBm for short) with Hurst parameter $H \in(0,1)$ is a centered Gaussian process $\left\{\beta^{H}(t), t \geq 0\right\}$ which is often used to model many complex phenomena in applications when the systems contain rough external forcing. When $H=1 / 2$, the $\mathrm{fBm}$ is the standard Brownian motion which is a Markov process and martingale, and we can use the classical Itô theory to construct a stochastic integration with respect to the standard Brownian motion. However, when $H \neq 1 / 2$, the $\mathrm{fBm}$ is neither a Markov process nor a semimartingale, and the $\mathrm{fBm}$ behaviors become quite different. The classical techniques based on PDE cannot apply to in this context. For more details on $\mathrm{fBm}$, we refer the readers to the articles [1-5].

Neutral differential equations (NDEs for short) are a family of differential equations depending on the past as well as the present state which involve derivatives with delays as well as the function itself. This family of equations has great applications in mathematical, electrical engineering, ecology, and other fields of science. In recent years, NDEs have received more and more attention; see, for example, [6$16]$ and the reference therein.

The observations of stock prices processes suggest that they are not self-similar. As a result, the mixed model describes the stock prices behavior in a better way. Since the pioneering paper by Cheridito [17], mixed stochastic models containing both a standard Brownian motion and $\mathrm{fBm}$ gained a lot of attention. The main reason for this is that they allow us to model systems driven by white noise and fractional Gaussian noise. For more details on this topic see [18-21].

The controllability problem is one of the most attractive and important problems of differential equations because of their great significance and applications in physics, population dynamics, engineering, mathematical biology, and other areas of science. Controllability for stochastic models only containing standard Brownian motion has been investigated very well (see [7, 12, 16, 22]). Recently, controllability for stochastic systems only driven by $\mathrm{fBm}$ has gained a lot of attention; we refer to $[6,9,11]$. Up to now, there is no paper which considers the controllability for stochastic systems driven by standard Brownian motion and $\mathrm{fBm}$.

In this paper, we study the existence, uniqueness, and controllability results for a class of neutral stochastic delay partial differential equations (NSDPDEs for short) driven by a standard Brownian motion and an $\mathrm{fBm}$ in the abstract form

$$
\begin{aligned}
& d[x(t)+h(t, x(t-r(t)))] \\
& =[A x(t)+f(t, x(t-\rho(t)))+B u(t)] d t \\
& \quad+g(t, x(t-\eta(t))) d W(t)+\sigma(t) d B_{\mathrm{Q}}^{H}(t), \\
& \quad t \geq 0, \\
& x(s)=\varphi(s) \in \mathscr{C}\left([-\tau, 0] ; \mathscr{L}^{2}(\Omega ; X)\right), \quad-\tau \leq s \leq 0,
\end{aligned}
$$


where $A$ is the infinitesimal generator of an analytic semigroup $\{S(t), t \geq 0\}$ of bounded linear operators in a real separable Hilbert space $X$. The delay functions $r, \rho, \eta:[0,+\infty) \rightarrow$ $[0, \tau](\tau \geq 0)$ are continuous, and $f, h:[0,+\infty) \times X \rightarrow X, g:$ $[0,+\infty) \times X \rightarrow \mathscr{L}_{\mathrm{Q}^{(1)}}^{0}\left(Y_{1} ; X\right)$, and $\sigma:[0,+\infty) \rightarrow \mathscr{L}_{\mathrm{Q}^{(2)}}^{0}\left(Y_{2} ; X\right)$ are Borel measurable satisfying appropriate conditions. The control mapping $u(\cdot)$ takes values in $\mathscr{L}^{2}([0, T] ; U)$, and the Hilbert space of admissible control mappings for a real separable Hilbert $U, B$ is a bounded linear operator from $U$ to $X .\{W(t), t \geq 0\}$ denotes a standard Brownian motion in a real separable Hilbert space $Y_{1} \cdot\left\{B_{Q}^{H}(t), t \geq 0\right\}$ denotes a fractional Brownian motion in a real separable Hilbert space $Y_{2}$ with Hurst parameter $H \in(1 / 2,1)$. Let $\mathscr{L}_{\mathrm{Q}^{(i)}}^{0}\left(Y_{i} ; X\right)(i=1,2)$ be the space of all $Q^{(i)}$-Hilbert-Schmidt operators from $Y_{i}$ into $X$.

Recently, Boufoussi and Hajji in [8] considered the existence and uniqueness problems of a class of neutral stochastic delay differential equations; that is, $B \equiv 0$ and $g \equiv 0$ in (1) by means of the Banach fixed point theory. Very recently, Liu and Luo in [13] studied the existence and uniqueness problems of a wide class of neutral stochastic delay partial differential equations, that is, $B \equiv 0$ in (1) by means of the Banach fixed point theory. However, they all required $1 / 2<\beta<1$ which is a stronger condition than ours (see Section 3$)$.

In this paper, based on the above papers, we also use the Banach fixed point theory to consider a class of neutral stochastic delay partial differential equations, which is more general than ones in $[8,13]$. Some conditions on the existence, uniqueness, and controllability of (1) are obtained. The related known results in Boufoussi and Hajji [8] and Liu and Luo in [13] are improved and generalized.

The contents of this paper are as follows. In Section 2, some notions, definitions, and lemmas which will be needed throughout this paper are introduced. In Section 3, the existence and uniqueness of mild solutions of (1) are proved. In Section 4, the controllability of (1) is investigated by means of the Banach fixed point theory. In Section 5, an example is given to illustrate our main results. At last, in Section 6, our conclusion is presented.

\section{Preliminaries}

Let $\left(\Omega, \mathscr{F},\left\{\mathscr{F}_{t}\right\}_{t \geq 0}, \mathbb{P}\right)$ be a complete probability space equipped with a normal filtration $\left\{\mathscr{F}_{t}\right\}_{t \geq 0}$ satisfying standard assumptions; that is, the filtration is right continuous and $\mathscr{F}_{0}$ contains all $\mathbb{P}$-null sets. Let $\mathscr{C}\left([-\tau, T] ; \mathscr{L}^{2}(\Omega ; X)\right)$ be the family of all continuous functions from $[-\tau, T]$ into $\mathscr{L}^{2}(\Omega ; X)$. Let $X$ and $Y_{i}, i=1,2$, be three real separable Hilbert spaces. We denote by $\mathscr{L}\left(Y_{i} ; X\right)$ the space of all bounded linear operators from $Y_{i}$ to $X, i=1,2$. We suppose that $\left\{e_{n}^{(i)}\right\}_{n \in \mathbb{N}^{+}}$is a complete orthonormal basis in $Y_{i}$. Let $Q^{(i)} \in$ $\mathscr{L}\left(Y_{i} ; X\right)$ be an operator defined by $Q^{(i)} e_{n}^{(i)}=\lambda_{n}^{(i)} e_{n}^{(i)}$ with finite trace $\operatorname{tr} Q^{(i)}=\sum_{n=1}^{\infty} \lambda_{n}^{(i)}<\infty$, where $\left\{\lambda_{n}^{(i)}\right\}_{n \in \mathbb{N}^{+}}$are nonnegative real numbers. Then there exists a real-valued sequence $\left\{\omega_{n}(t)\right\}_{n \in \mathbb{N}^{+}}$of one-dimensional standard Brownian motions mutually independent on $\left(\Omega, \mathscr{F}_{,},\left\{\mathscr{F}_{t}\right\}_{t \geq 0}, \mathbb{P}\right)$ such that

$$
W(t)=\sum_{n=1}^{\infty} \sqrt{\lambda_{n}^{(1)}} \omega_{n}(t) e_{n}^{(1)}, \quad t \geq 0 .
$$

Consider that a $Y_{2}$-valued stochastic process $B_{Q}^{H}(t)$ is defined by the formal infinite sum (see [2]).

$$
B_{\mathrm{Q}}^{H}(t)=\sum_{n=1}^{\infty} \sqrt{\lambda_{n}^{(2)}} \beta_{n}^{H}(t) e_{n}^{(2)}, \quad t \geq 0,
$$

where the sequence $\left\{\beta_{n}^{H}(t)\right\}_{n \in \mathbb{N}^{+}}$is mutually independent scalar fBms with Hurst parameter $H \in(1 / 2,1)$.

Let $\mathscr{L}_{\mathrm{Q}^{(i)}}^{0}\left(Y_{i} ; X\right)$ be the space of all $Q^{(i)}$-Hilbert-Schmidt operators from $Y_{i}$ to $X, i=1,2$. Now we show the following definitions.

Definition 1. Let $\xi_{i} \in \mathscr{L}\left(Y_{i} ; X\right)$ and define

$$
\left\|\xi_{i}\right\|_{\mathscr{L}_{Q^{(i)}}^{0}}:=\operatorname{tr}\left(\xi_{i} Q^{(i)} \xi_{i}^{*}\right)=\left\{\sum_{n=1}^{\infty}\left\|\sqrt{\lambda_{n}^{(i)}} \xi_{i} e_{n}^{(i)}\right\|_{X}^{2}\right\}^{1 / 2} .
$$

If $\left\|\xi_{i}\right\|_{\mathscr{L}_{\mathrm{Q}^{(i)}}}<\infty$, then $\xi_{i}$ is called a $Q^{(i)}$-Hilbert-Schmidt operator and the space $\mathscr{L}_{\mathrm{Q}^{(i)}}^{0}:=\mathscr{L}_{\mathrm{Q}^{(i)}}^{0}\left(Y_{i} ; X\right)$ is a real separable Hilbert space equipped with the inner product $\langle\varphi, \psi\rangle_{\mathscr{L}_{Q^{(i)}}^{0}}=$ $\sum_{n=1}^{\infty}\left\langle\varphi e_{n}^{(i)}, \psi e_{n}^{(i)}\right\rangle, i=1,2$.

Let $T>0, \beta=\{\beta(t) ; t \in[0, T]\}$ be a Wiener process and $\left\{\beta^{H}(t), t \in[0, T]\right\}$ be the one-dimensional $\mathrm{fBm}$ with Hurst parameter $H \in(1 / 2,1)$. $\beta^{H}$ has the following stochastic integral representation:

$$
\beta^{H}(t)=\int_{0}^{t} K_{H}(t, s) d \beta(s),
$$

$K_{H}(t, s)$ is the kernel defined as

$$
K_{H}(t, s)=c_{H} s^{1 / 2-H} \int_{s}^{t}(u-s)^{H-3 / 2} u^{H-1 / 2} d u
$$

for $t>s$, where $c_{H}=(H(2 H-1) / B(2-2 H, H-1 / 2))^{1 / 2}$, and $B(\cdot, \cdot)$ denotes the Beta function. Set $K_{H}(t, s)=0$ for $t \leq$ $s$. Let $\mathscr{H}$ denote the reproducing kernel Hilbert space of the $\mathrm{fBm}$. In fact $\mathscr{H}$ is the closure of the set of indicator functions $\left\{I_{[0 ; t]}, t \in[0, T]\right\}$ with respect to the inner product.

$$
\left\langle I_{[0, t]}, I_{[0, s]}\right\rangle_{\mathscr{C}}=R_{H}(t, s) .
$$

Then the mapping $I_{[0, t]} \rightarrow \beta^{H}(t)$ can be extended to an isometry between $\mathscr{H}$ and the first Wiener chaos of the $\mathrm{fBm}$ $\overline{\operatorname{span}}^{\mathscr{L}^{2}(\Omega)}\left\{\beta^{H}, t \in[0, T]\right\}$. The image of an element $\varphi \in \mathscr{H}$ by this isometry is called the Wiener integral of $\varphi$ with respect to $\beta^{H}$ (see [23]).

We recall that for $\psi, \varphi \in \mathscr{H}$ their inner product in $\mathscr{H}$ is given by

$$
\begin{aligned}
& \langle\psi, \varphi\rangle_{\mathscr{H}} \\
& \quad=H(2 H-1) \int_{0}^{T} \int_{0}^{T} \psi(s) \varphi(t)|t-s|^{2 H-2} d s d t .
\end{aligned}
$$


$K_{H}^{*}$ is an operator from $\mathscr{H}$ to $\mathscr{L}^{2}([0, T])$ defined as

$$
\left(K_{H}^{*} \varphi\right)(s)=\int_{s}^{t} \varphi(r) \frac{\partial K_{H}(r, s)}{\partial r} d r
$$

Then

$$
\left(K_{H}^{*} I_{[0, t]}\right)(s)=K_{H}(t, s) I_{[0, t]}(s)
$$

and $K_{H}^{*}$ is an isometry between the set of indicator functions $\left\{I_{[0 ; t]}, t \in[0, T]\right\}$ and $\mathscr{L}^{2}([0, T])$ that can be extended to $\mathscr{H}$ (see [1]).

Let $\psi(s)$ for $s \in[0, T]$ be a mapping with values in $\mathscr{L}_{2}^{0}\left(Y_{2} ; X\right)$. The stochastic integral of $\psi$ with respect to $B_{\mathrm{Q}}^{H}$ is defined by

$$
\begin{aligned}
\int_{0}^{t} \psi(s) d B_{\mathrm{Q}}^{H}(s) & =\sum_{n=1}^{\infty} \int_{0}^{t} \sqrt{\lambda_{n}^{(2)}} \psi(s) e_{n}^{(2)} d \beta_{n}^{H}(s) \\
& =\sum_{n=1}^{\infty} \int_{0}^{t} \sqrt{\lambda_{n}^{(2)}} K_{H}^{*}\left(\psi e_{n}^{(2)}\right)(s) d \beta_{n}(s),
\end{aligned}
$$

where $\beta_{n}$ is the standard Brownian motion which was introduced in (5).

In order to set existence, uniqueness, and controllability problems, we need the following lemmas which are Lemma 2 in [2] and Lemma 7.7 in [24], respectively.

Lemma 2 (see [2]). For any $\varphi:[0, T] \mapsto \mathscr{L}_{\mathrm{Q}^{(2)}}^{0}\left(Y_{2} ; X\right)$ and $0 \leq a \leq b \leq T$, if $\sum_{n=1}^{\infty} \sqrt{\lambda_{n}^{(2)}}<\infty$ and the series $\sum_{n=1}^{\infty} \sqrt{\lambda_{n}^{(2)}} \varphi(t) e_{n}^{(2)}$ is uniformly convergent for $t \in[0, T]$, then one has

$$
\begin{aligned}
& \mathbb{E}\left\|\int_{a}^{b} \varphi(s) d B_{Q}^{H}(s)\right\|_{X}^{2} \\
& \quad \leq c_{H} H(2 H-1)(b-a)^{2 H-1} \int_{a}^{b}\|\varphi(s)\|_{\mathscr{L}^{0}(2)}^{2} d s,
\end{aligned}
$$

where $H \quad \in \quad(1 / 2,1)$ and $c_{H}$ $\sqrt{H(2 H-1) / B(2-2 H, H-1 / 2)}$.

Lemma 3 (see [24]). For any $r \geq 1$ and for arbitrary predictable process $\Phi(\cdot) \in \mathscr{L}_{\mathrm{Q}^{(1)}}^{0}\left(Y_{1} ; X\right)$, one has

$$
\begin{aligned}
& \sup _{s \in[0, t]} \mathbb{E}\left|\int_{0}^{s} \phi(\sigma) d W(\sigma)\right|^{2 r} \\
& \leq(r(2 r-1))^{r}\left(\int_{0}^{t}\left(\mathbb{E}\|\phi(s)\|_{\mathscr{L}_{\mathrm{Q}^{(1)}}^{0}}^{2 r}\right)^{1 / r} d s\right)^{r} \\
& t \in[0, T] .
\end{aligned}
$$

We assume that $0 \in \rho(A)$, where $\rho(A)$ is the resolvent set of $A$ and the analytic semigroup $\{S(t), t \geq 0\}$ in $X$ is uniformly bounded; that is, $\|S(t)\|_{X} \leq M$ for some positive constant $M \geq 1$. Then, for $\alpha \in(0,1]$, it is possible under some circumstance to define the fractional power $(-A)^{\alpha}$ as a closed linear operator with domain $D\left((-A)^{\alpha}\right)$. Moreover, the subspace $D\left((-A)^{\alpha}\right)$ is dense in $X$ and the expression

$$
\|v\|_{\alpha}=\left\|(-A)^{\alpha} v\right\|_{X}, \quad v \in D\left((-A)^{\alpha}\right)
$$

defines a norm on $D\left((-A)^{\alpha}\right)$.

Let $H_{\alpha}$ denote the Banach space $D\left((-A)^{\alpha}\right)$ equipped with the norm $\|\cdot\|_{\alpha}$, then the following properties are well known (see [25]).

Lemma 4 (see [25]). Suppose that the preceding conditions are satisfied.

(1) If $0<\beta \leq \alpha$, then the injection $H_{\alpha} \hookrightarrow H_{\beta}$ is continuous.

(2) For every $0<\beta \leq 1$, there exists $M_{\beta}>0$ such that

$$
\left\|(-A)^{\beta} S(t)\right\| \leq M_{\beta} t^{-\beta}, \quad 0 \leq t \leq T .
$$

\section{Existence and Uniqueness of Mild Solution}

In this section, the Banach fixed point theory is used to investigate the existence and uniqueness problems of (1). Now, we introduce the definition of mild solution of (1).

Definition 5. An $X$-valued stochastic process $x(t)$ is called a mild solution of $(1)$ if $x(\cdot) \in C\left([-\tau, T] ; \mathscr{L}^{2}(\Omega ; X)\right)$ and $x(t)=$ $\varphi(t)$ for $t \in[-\tau, 0]$ and for $t \in[0, T]$ satisfies

$$
\begin{aligned}
x(t)= & S(t)[\varphi(0)+h(0, x(-r(0)))] \\
& -h(t, x(t-r(t))) \\
& -\int_{0}^{t} A S(t-s) h(s, x(s-r(s))) d s \\
& +\int_{0}^{t} S(t-s)[f(s, x(s-\rho(s)))+B u(s)] d s \\
& +\int_{0}^{t} S(t-s) g(s, x(s-\eta(s))) d W(s) \\
& +\int_{0}^{t} S(t-s) \sigma(s) d B_{\mathrm{Q}}^{H}(s) \quad \mathbb{P} \text {-a.s. }
\end{aligned}
$$

In order to set the existence and uniqueness problems, we assume that the following conditions hold:

(C.1) $A$ is the infinitesimal generator of an analytic semigroup $\{S(t), t \geq 0\}$ of bounded linear operators in $X$ and there exists a positive constant $M \geq 1$ such that $\|S(t)\|_{X} \leq M$ for $0 \leq t \leq T$.

(C.2) The mappings $f:[0,+\infty) \times X \rightarrow X$ and $g$ : $[0,+\infty) \times X \rightarrow \mathscr{L}_{\mathrm{Q}^{(1)}}^{0}\left(Y_{1} ; X\right)$ satisfy the following conditions: for any $x, y \in X$ and $t \in[0, T]$, there exist nonnegative constants $L_{f}, C_{f}, L_{g}$, and $C_{g}$, such that

(i) $\|f(t, x)-f(t, y)\|_{X} \leq L_{f}\|x-y\|_{X},\|f(t, x)\|_{X}^{2} \leq$ $C_{f}\left(1+\|x\|_{X}^{2}\right)$

(ii) $\|g(t, x)-g(t, y)\|_{\mathscr{L}_{\mathrm{Q}^{(1)}}} \leq L_{g}\|x-y\|_{X}$, $\|g(t, x)\|_{\mathscr{L}_{\mathrm{Q}^{(1)}}}^{2} \leq C_{g}\left(1+\|x\|_{X}^{2}\right)$. 
(C.3) The mapping $h(t, x) \in \mathscr{D}\left((-A)^{\beta}\right)$ satisfies the following conditions: for any $x, y \in X$ and $t \geq 0$, there exist nonnegative constants $L_{h}, C_{h} \geq 0$ and $\beta \in(0,1)$ such that

(i) $\left\|(-A)^{\beta} h(t, x)-(-A)^{\beta} h(t, y)\right\|_{X} \leq L_{h}\|x-y\|_{X}$;

(ii) $\left\|(-A)^{\beta} h(t, x)\right\|_{X}^{2} \leq C_{h}\left(1+\|x\|_{X}^{2}\right)$;

(iii) the constants $L_{h}$ and $\beta$ satisfy the following inequality $k:=L_{h}\left\|(-A)^{-\beta}\right\|<1$.

(C.4) The mapping $(-A)^{\beta} h$ is continuous in mean square: for all $x \in C\left([0, T] ; \mathscr{L}^{2}(\Omega ; X)\right)$, $\lim _{t \rightarrow s} \mathbb{E}\left\|(-A)^{\beta} h(t, x(t))-(-A)^{\beta} h(s, x(s))\right\|_{X}^{2}=0$.

(C.5) The mapping $\sigma:[0, T] \rightarrow \mathscr{L}_{\mathrm{Q}^{(2)}}^{0}\left(Y_{2} ; X\right)$ satisfies $\int_{0}^{T}\|\sigma(s)\|_{\mathscr{L}_{Q^{(2)}}^{0}}^{2} d s<\infty, \forall T>0$.

(C.6) The mapping $u(\cdot)$ takes values in $\mathscr{L}^{2}([0, T] ; U)$ and $B$ is a bounded linear operator from $U$ to $X$.

Theorem 6. Let conditions (C.1)-(C.6) be satisfied. Then system (1) has a unique mild solution on $[-\tau, T]$.

Proof. Denote $\mathscr{B}_{T}:=C\left([-\tau, T] ; \mathscr{L}^{2}(\Omega ; X)\right)$ by subspace of all continuous functions form $[-\tau, T]$ into $\mathscr{L}^{2}(\Omega ; X)$ equipped with the norm $\|\zeta\|_{\mathscr{B}_{T}}=\sup _{s \in[-\tau, T]}\left(\mathbb{E}\|\zeta(s)\|^{2}\right)^{1 / 2}$. Fix $T>0$ and consider the space $S_{T}:=\left\{x \in \mathscr{B}_{T}: x(s)=\varphi(s)\right.$, for $s \in[-\tau, 0]\} . S_{T}$ is a closed subspace of $\mathscr{B}_{T}$ equipped with the norm $\|\cdot\|_{\mathscr{B}_{T}}$. Obviously, $S_{T}$ and $\mathscr{B}_{T}$ are two Banach spaces. Define an operator $\pi: S_{T} \rightarrow S_{T}$ by $\pi(x)(t)=\varphi(t)$ for $t \epsilon$ $[-\tau, 0]$ and for $t \in[0, T]$,

$$
\begin{aligned}
\pi(x)(t) & \\
= & S(t)[\varphi(0)+h(0, x(-r(0)))] \\
& -h(t, x(t-r(t))) \\
& -\int_{0}^{t} A S(t-s) h(s, x(s-r(s))) d s \\
& +\int_{0}^{t} S(t-s)[f(s, x(s-\rho(s)))+B u(s)] d s \\
& +\int_{0}^{t} S(t-s) g(s, x(s-\eta(s))) d W(s) \\
& +\int_{0}^{t} S(t-s) \sigma(s) d B_{\mathrm{Q}}^{H}(s) .
\end{aligned}
$$

We first verify that $\pi$ is continuous in mean square on $[0, T]$. Let $x \in S_{T}, \quad 0 \leq t<T$, and let $\varepsilon$ be positive and sufficiently small (similar estimates hold for $\varepsilon<0$ ), then

$$
\begin{aligned}
\mathbb{E}\|\pi(x)(t+\varepsilon)-\pi(x)(t)\|_{X}^{2} \leq 7 \mathbb{E} \|(S(t+\varepsilon)-S(t)) \\
\cdot[\varphi(0)+h(0, x(-r(0)))] \|_{X}^{2} \\
+7 \mathbb{E} \| h(t+\varepsilon, x(t+\varepsilon-r(t+\varepsilon)))
\end{aligned}
$$

$$
\begin{aligned}
& -h(t, x(t-r(t))) \|_{X}^{2} \\
& +7 \mathbb{E} \| \int_{0}^{t+\varepsilon} A S(t+\varepsilon-s) h(s, x(s-r(s))) d s \\
& -\int_{0}^{t} A S(t-s) h(s, x(s-r(s))) d s \|_{X}^{2} \\
& +7 \mathbb{E} \| \int_{0}^{t+\varepsilon} S(t+\varepsilon-s) f(s, x(s-\rho(s))) d s \\
& -\int_{0}^{t} S(t-s) f(s, x(s-\rho(s))) d s \|_{X}^{2} \\
& +7 \mathbb{E} \| \int_{0}^{t+\varepsilon} S(t+\varepsilon-s) g(s, x(s-\eta(s))) d W(s) \\
& -\int_{0}^{t} S(t-s) g(s, x(s-\eta(s))) d W(s) \|_{X}^{2} \\
& +7 \mathbb{E} \| \int_{0}^{t+\varepsilon} S(t+\varepsilon-s) \sigma(s) d B_{\mathrm{Q}}^{H}(s) \\
& -\int_{0}^{t} S(t-s) \sigma(s) d B_{\mathrm{Q}}^{H}(s) \|_{X}^{2} \\
& +7 \mathbb{E} \| \int_{0}^{t+\varepsilon} S(t+\varepsilon-s) B u(s) d s \\
& -\int_{0}^{t} S(t-s) B u(s) d s\left\|_{X}^{2}:=7 \sum_{i=1}^{7} \mathbb{E}\right\| K_{i}(\varepsilon) \|_{X}^{2} .
\end{aligned}
$$

It is easy to know that $\mathbb{E}\left\|K_{i}(\varepsilon)\right\|_{X}^{2} \rightarrow 0$ as $\varepsilon \rightarrow 0, i=1,2,4,7$. Further, by using Cauchy-Schwarz inequality, we get

$$
\begin{gathered}
\mathbb{E}\left\|K_{3}(\varepsilon)\right\|_{X}^{2} \leq 2 \mathbb{E} \| \int_{0}^{t}(S(\varepsilon)-I)(-A)^{1-\beta} S(t-s) \\
\cdot(-A)^{\beta} h(s, x(s-r(s))) d s\left\|_{X}^{2}+2 \mathbb{E}\right\| \int_{t}^{t+\varepsilon}(-A)^{1-\beta} \\
\cdot S(t+\varepsilon-s)(-A)^{\beta} h(s, x(s-r(s))) d s \|_{X}^{2} \\
:=2 \mathbb{E}\left\|K_{31}(\varepsilon)\right\|_{X}^{2}+2 \mathbb{E}\left\|K_{32}(\varepsilon)\right\|_{X}^{2} .
\end{gathered}
$$

Applying conditions (C.1) and (C.3) to $\mathbb{E}\left\|K_{31}(\varepsilon)\right\|_{X}^{2}$, we can obtain

$$
\begin{aligned}
& \mathbb{E}\left\|K_{31}(\varepsilon)\right\|_{U}^{2} \leq(S(\varepsilon)-I)^{2} \\
& \cdot M_{1-\beta}^{2} \mathbb{E} \| \int_{0}^{t} \frac{1}{(t-s)^{1-\beta}}(-A)^{\beta} \\
& \cdot h(s, x(s-r(s))) d s \|_{X}^{2} \leq(S(\varepsilon)-I)^{2} \\
& \cdot M_{1-\beta}^{2} T^{\beta} \beta^{-1} \int_{0}^{t} \frac{1}{(t-s)^{1-\beta}} \mathbb{E} \|(-A)^{\beta} \\
& \cdot h(s, x(s-r(s))) \|_{X}^{2} d s \leq(S(\varepsilon)-I)^{2} \\
& \cdot M_{1-\beta}^{2} T^{2 \beta} \beta^{-2} C_{h}\left(1+\sup _{s \in[-\tau, T]} \mathbb{E}\|x(s)\|_{X}^{2}\right) \longrightarrow 0
\end{aligned}
$$


as $\varepsilon \rightarrow 0$. Applying condition (C.1) and (C.3) to $\mathbb{E}\left\|I_{32}(\varepsilon)\right\|_{X}^{2}$, we have

$$
\begin{aligned}
& \mathbb{E}\left\|K_{32}(\varepsilon)\right\|_{U}^{2} \leq M_{1-\beta}^{2} \mathbb{E} \| \int_{t}^{t+\varepsilon} \frac{1}{(t+\varepsilon-s)^{1-\beta}}(-A)^{\beta} \\
& \cdot h(s, x(s-r(s))) d s \|_{X}^{2} \\
& \leq M_{1-\beta^{2}}^{2} \varepsilon^{\beta} \int_{t}^{t+\varepsilon} \frac{1}{(t+\varepsilon-s)^{1-\beta}} \mathbb{E} \|(-A)^{\beta} \\
& \cdot h(s, x(s-r(s))) \|_{X}^{2} d s \leq M_{1-\beta}^{2} \varepsilon^{2 \beta} \beta^{-2} C_{h}(1 \\
& \left.+\sup _{s \in[-\tau, T]} \mathbb{E}\|x(s)\|_{X}^{2}\right) \longrightarrow 0
\end{aligned}
$$

as $\varepsilon \rightarrow 0$. That is to say, $\mathbb{E}\left\|K_{3}(\varepsilon)\right\|_{U}^{2} \rightarrow 0$ as $\varepsilon \rightarrow 0$.

For the fifth term, by using Lemma 3 , we can obtain

$$
\begin{aligned}
& \mathbb{E}\left\|K_{5}(\varepsilon)\right\|_{X}^{2} \leq 2 \mathbb{E} \| \int_{0}^{t}(S(\varepsilon)-I) S(t-s) \\
& \cdot g(s, x(s-\eta(s))) d W(s) \|_{X}^{2} \\
& +2 \mathbb{E} \| \int_{t}^{t+\varepsilon} S(t+\varepsilon-s) \\
& \cdot g(s, x(s-\eta(s))) d W(s) \|_{X}^{2} \leq 2(S(\varepsilon) \\
& -I)^{2} \int_{0}^{t} \mathbb{E}\|S(t-s) g(s, x(s-\eta(s)))\|_{\mathscr{L}_{Q^{(1)}}^{0}}^{2} d s \\
& +2 \int_{t}^{t+\varepsilon} \mathbb{E}\|S(t+\varepsilon-s) g(s, x(s-\eta(s)))\|_{\mathscr{L}_{Q^{(1)}}^{0}}^{2} d s \longrightarrow
\end{aligned}
$$

0

as $\varepsilon \rightarrow 0$.

For the sixth term, we get

$$
\begin{aligned}
& \mathbb{E}\left\|K_{6}(\varepsilon)\right\|_{X}^{2} \\
& \leq 2 \mathbb{E}\left\|\int_{0}^{t}(S(t+\varepsilon-s)-S(t-s)) \sigma(s) d B_{Q}^{H}(s)\right\|_{X}^{2} \\
& \quad+2 \mathbb{E}\left\|\int_{t}^{t+\varepsilon} S(t+\varepsilon-s) \sigma(s) d B_{Q}^{H}(s)\right\|_{X}^{2} \\
& :=2 \mathbb{E}\left\|K_{61}(\varepsilon)\right\|_{X}^{2}+2 \mathbb{E}\left\|K_{62}(\varepsilon)\right\|_{X}^{2} .
\end{aligned}
$$

Firstly, apply Lemma 2 to $\mathbb{E}\left\|K_{61}(\varepsilon)\right\|_{X}^{2}$, and we have

$$
\begin{aligned}
\mathbb{E} & \left\|K_{61}(\varepsilon)\right\|_{X}^{2} \leq c_{H} H(2 H-1) \\
\cdot & T^{2 H-1} \int_{0}^{t}\|S(t-s)(S(\varepsilon)-I) \sigma(s)\|_{\mathscr{L}_{\mathrm{Q}^{(2)}}}^{2} d s \\
\leq & (S(\varepsilon)-I)^{2} c_{H} H(2 H-1) \\
\cdot & T^{2 H-1} M^{2} \int_{0}^{t}\|\sigma(s)\|_{\mathscr{L}_{\mathrm{Q}^{(2)}}^{0}}^{2} d s \longrightarrow 0
\end{aligned}
$$

as $\varepsilon \rightarrow 0$. For every $s$ fixed, we get $S(\varepsilon) \sigma(s) \rightarrow \sigma(s)$ as $\varepsilon \rightarrow 0$ and $\|S(\varepsilon) \sigma(s)\|_{\mathscr{L}_{\mathrm{Q}^{(2)}}^{0}} \leq M\|\sigma(s)\|_{\mathscr{L}_{\mathrm{Q}^{(2)}}^{0}}$.
By Lemma 2 and the Lebesgue dominated convergence theorem, we can obtain

$$
\begin{aligned}
& \mathbb{E}\left\|K_{62}(\varepsilon)\right\|_{X}^{2} \\
& \quad \leq c_{H} H(2 H-1) \varepsilon^{2 H-1} M^{2} \int_{t}^{t+\varepsilon}\|\sigma(s)\|_{\mathscr{L}^{0}(2)}^{2} d s \longrightarrow
\end{aligned}
$$

0

as $\varepsilon \rightarrow 0$. Therefore $\mathbb{E}\left\|K_{6}(\varepsilon)\right\|_{X}^{2} \rightarrow 0$ as $\varepsilon \rightarrow 0$. Thus, $\pi$ is continuous in mean square on $[0, T]$. So we conclude that $\pi\left(S_{T}\right) \subset S_{T}$.

Moreover, we shall show that $\pi$ is contractive in $S_{T_{1}}$ with some $0<T_{1} \leq T$. Let $x, y \in S_{T}$ and for any fixed $t \in[0, T]$, we get

$$
\begin{aligned}
& \mathbb{E}\|\pi(x)(t)-\pi(y)(t)\|_{X}^{2} \leq \frac{1}{k} \mathbb{E} \| h(t, x(t-r(t))) \\
& -h(t, y(t-r(t)))^{2}\left\|_{X}+\frac{3}{1-k} \mathbb{E}\right\| \int_{0}^{t}(-A) S(t-s) \\
& \cdot[h(s, x(s-r(s)))-h(s, y(s-r(s)))] d s \|_{X}^{2} \\
& +\frac{3}{1-k} \mathbb{E} \| \int_{0}^{t} S(t-s)[f(s, x(s-\rho(s))) \\
& -f(s, y(s-\rho(s)))] d s\left\|_{X}^{2}+\frac{3}{1-k} \mathbb{E}\right\| \int_{0}^{t} S(t \\
& -s)[g(s, x(s-\eta(s))) \\
& -g(s, y(s-\eta(s)))] d W(s) \|_{X}^{2}
\end{aligned}
$$

By conditions (C.1)-(C.4) and Lemma 3, we have

$$
\begin{aligned}
\mathbb{E}\|\pi(x)(t)-\pi(y)(t)\|^{2} \\
\leq k \mathbb{E}\|x(t-r(t))-y(t-r(t))\|_{X}^{2} \\
+\frac{3 M_{1-\beta}^{2} L_{h}^{2} t^{2 \beta}}{(1-k) \beta^{2}} \sup _{s \in[-\tau, T]} \mathbb{E}\|x(s)-y(s)\|_{X}^{2} \\
+\frac{3 M^{2} L_{f}^{2} t^{2}}{1-k} \sup _{s \in[-\tau, T]} \mathbb{E}\|x(s)-y(s)\|_{X}^{2} \\
+\frac{3 M^{2} L_{g}^{2} t}{1-k} \sup _{s \in[-\tau, T]} \mathbb{E}\|x(s)-y(s)\|_{X}^{2} .
\end{aligned}
$$

Hence

$$
\begin{aligned}
& \sup _{t \in[-\tau, T]} \mathbb{E}\|\pi(x)(t)-\pi(y)(t)\|_{X}^{2} \\
& \quad \leq \gamma(t) \sup _{t \in[-\tau, T]} \mathbb{E}\|x(t)-y(t)\|_{X}^{2},
\end{aligned}
$$

where

$$
\gamma(t)=k+\frac{3 M_{1-\beta}^{2} L_{h}^{2} t^{2 \beta}}{(1-k) \beta^{2}}+\frac{3 M^{2} L_{f}^{2} t^{2}}{1-k}+\frac{3 M^{2} L_{g}^{2} t}{1-k} .
$$

By condition (C.3), we have $\gamma(0)=k<1$, then there exists $0<T_{1} \leq T$ such that $0<\gamma\left(T_{1}\right)<1$ which shows that 
$\pi$ is a contraction mapping in $S_{T_{1}}$; therefore $\pi$ has a unique fixed point in $S_{T_{1}}$, which is a unique mild solution of (1) on $\left[-\tau, T_{1}\right]$. Repeat this procedure to extend the mild solution to $[\tau, T]$. The proof is completed.

Remark 7. Boufoussi and Hajji in [8] and Liu and Luo in [13], respectively, considered the existence and uniqueness of solutions for special cases of (1). In (20) and (21), we use the Hölder inequality and linear growth condition to obtain the continuity of $K_{3}(\varepsilon)$ and obtain a weaker result than those of $[8,13]$. The necessary conditions both in $[8,13]$ are

$$
1 / 2<\beta<1 \text {. }
$$

However, our condition is

$$
0<\beta<1 \text {. }
$$

In this sense, this paper improves and generalizes the results in $[8,13]$.

\section{Controllability Result}

In this section, we focus on the controllability problem of (1). Now we introduce the concept of controllability of neutral stochastic delay partial differential equations.

Definition 8. System (1) is said to be controllable on the finite interval $[-\tau, T]$, if, for each initial stochastic process $\varphi$ defined on $[-\tau, 0]$ and $x_{1} \in X$, there exists a stochastic control $u \in \mathscr{L}^{2}([0, T] ; U)$ which is adapted to the filtration $\left\{\mathscr{F}_{t}\right\}_{t \geq 0}$ such that the mild solution $x(\cdot)$ of (1) satisfies $x(T)=x_{1}$, where $x_{1}$ and $T$ are the preassigned terminal state and time, respectively.

In order to set the controllability problem, we assume that the following conditions hold:

(C.7) The linear operator $\omega$ from $U$ to $X$ defined by $\omega u=$ $\int_{0}^{T} S(T-s) B u(s) d s$ has an inverse operator $\omega^{-1}$ with values in $\mathscr{L}^{2}([0, T] ; U) \backslash \operatorname{ker} \omega$, where $\operatorname{ker} \omega=\{x \in$ $\left.\left.\mathscr{L}^{2}([0, T]) ; U\right): \omega x=0\right\}$ (see $\left.[22]\right)$, and there exists a pair of finite positive constants $M_{B}$ and $M_{\omega}$ such that $\|B\| \leq M_{B}$ and $\left\|\omega^{-1}\right\| \leq M_{\omega}$.

Theorem 9. Let conditions (C.1)-(C.7) be satisfied. Then, system (1) is controllable on $[-\tau, T]$.

Proof. Using the condition (C.7) for an arbitrary mapping $x(\cdot)$, define the stochastic control by

$$
\begin{aligned}
& u(t)=\omega^{-1}\left\{x_{1}-S(T)[\varphi(0)+h(0, x(-r(0)))]\right. \\
& +h(T, x(T-r(T))) \\
& \quad+\int_{0}^{T} A S(T-s) h(s, x(s-r(s))) d s \\
& \quad-\int_{0}^{T} S(T-s) f(s, x(s-\rho(s))) d s \\
& \quad-\int_{0}^{T} S(T-s) g(s, x(s-\eta(s))) d W(s) \\
& \left.\quad-\int_{0}^{T} S(T-s) \sigma(s) d B_{\mathrm{Q}}^{H}(s)\right\}(t), \quad t \in[0, T] .
\end{aligned}
$$

Applying this control to the operator $\pi$. Obviously, to find a fixed point for the operator $\pi$ is equivalent to prove the existence of mild solutions of equation. Clearly, $\pi(T)=x_{1}$, which implies that the stochastic control $u$ steers the system from the initial state $\varphi$ to $x_{1}$ in time $T$, provided we can find a fixed point of the operator $\pi$ which means that the system is controllable on $[-\tau, T]$.

First, we shall prove that $\pi$ is continuous in mean square on $[0, T]$. Let $x \in S_{T}, 0 \leq t<T$, and $\varepsilon$ be positive and sufficiently small (similar estimates hold for $\varepsilon<0$ ), then

$$
\begin{aligned}
& \mathbb{E}\|\pi(x)(t+\varepsilon)-\pi(x)(t)\|_{X}^{2} \leq 7 \mathbb{E} \|(S(t+\varepsilon)-S(t)) \\
& \cdot[\varphi(0)+h(0, x(-r(0)))]\left\|_{X}^{2}+7 \mathbb{E}\right\| h(t+\varepsilon, x(t \\
& +\varepsilon-r(t+\varepsilon)))-h(t, x(t-r(t))) \|_{X}^{2} \\
& +7 \mathbb{E} \| \int_{0}^{t+\varepsilon} A S(t+\varepsilon-s) h(s, x(s-r(s))) d s \\
& -\int_{0}^{t} A S(t-s) h(s, x(s-r(s))) d s \|_{X}^{2} \\
& +7 \mathbb{E} \| \int_{0}^{t+\varepsilon} S(t+\varepsilon-s) f(s, x(s-\rho(s))) d s \\
& -\int_{0}^{t} S(t-s) f(s, x(s-\rho(s))) d s \|_{X}^{2} \\
& +7 \mathbb{E} \| \int_{0}^{t+\varepsilon} S(t+\varepsilon-s) g(s, x(s-\eta(s))) d W(s) \\
& -\int_{0}^{t} S(t-s) g(s, x(s-\eta(s))) d W(s) \|_{X}^{2} \\
& +7 \mathbb{E} \| \int_{0}^{t+\varepsilon} S(t+\varepsilon-s) \sigma(s) d B_{\mathrm{Q}}^{H}(s)-\int_{0}^{t} S(t-s) \\
& \cdot \sigma(s) d B_{Q}^{H}(s)\left\|_{X}^{2}+7 \mathbb{E}\right\| \int_{0}^{t+\varepsilon} S(t \\
& +\varepsilon-v) B \omega^{-1}\left\{x_{1}-S(T)[\varphi(0)+h(0, x(-r(0)))]\right. \\
& +h(T, x(T-r(T))) \\
& +\int_{0}^{T} A S(T-s) h(s, x(s-r(s))) d s \\
& -\int_{0}^{T} S(T-s) f(s, x(s-\rho(s))) d s \\
& -\int_{0}^{T} S(T-s) g(s, x(s-\eta(s))) d W(s) \\
& \left.-\int_{0}^{T} S(T-s) \sigma(s) d B_{\mathrm{Q}}^{H}(s)\right\} d v-\int_{0}^{t} S(t \\
& \text {-v) } B \omega^{-1}\left\{x_{1}-S(T)[\varphi(0)+h(0, x(-r(0)))]\right. \\
& +h(T, x(T-r(T))) \\
& +\int_{0}^{T} A S(T-s) h(s, x(s-r(s))) d s \\
& -\int_{0}^{T} S(T-s) f(s, x(s-\rho(s))) d s
\end{aligned}
$$




$$
\begin{aligned}
& -\int_{0}^{T} S(T-s) g(s, x(s-\eta(s))) d W(s) \\
& \left.-\int_{0}^{T} S(T-s) \sigma(s) d B_{\mathrm{Q}}^{H}(s)\right\} d v \|_{X}^{2} \\
& :=7 \sum_{i=1}^{7} \mathbb{E}\left\|K_{i}(\varepsilon)\right\|_{X}^{2} .
\end{aligned}
$$

From Section 3, we can obtain that $\mathbb{E}\left\|K_{i}(\varepsilon)\right\|_{X}^{2} \rightarrow 0$ as $\varepsilon \rightarrow 0$, $i=1,2, \ldots, 6$. Now we estimate the term $\mathbb{E}\left\|K_{7}(\varepsilon)\right\|_{X}^{2}$, and we have

$$
\begin{aligned}
\mathbb{E} & \left\|K_{7}(\varepsilon)\right\|_{X}^{2} \leq 2 \mathbb{E} \| \int_{t}^{t+\varepsilon} S(t+\varepsilon-v) B \omega^{-1}\left\{x_{1}\right. \\
& -S(T)[\varphi(0)+h(0, x(-r(0)))] \\
& +h(T, x(T-r(T))) \\
& +\int_{0}^{T} A S(T-s) h(s, x(s-r(s))) d s \\
& -\int_{0}^{T} S(T-s) f(s, x(s-\rho(s))) d s \\
& -\int_{0}^{T} S(T-s) g(s, x(s-\eta(s))) d W(s) \\
& \left.-\int_{0}^{T} S(T-s) \sigma(s) d B_{Q}^{H}(s)\right\} d v \|_{X}^{2} \\
& +2 \mathbb{E} \| \int_{0}^{t}(S(t+\varepsilon-s)-S(t-s)) B \omega^{-1}\left\{x_{1}\right. \\
& -S(T)[\varphi(0)+h(0, x(-r(0)))] \\
& +h(T, x(T-r(T))) \\
& +\int_{0}^{T} A S(T-s) h(s, x(s-r(s))) d s \\
& \left.-\int_{0}^{T} S(T-s) \sigma(s) d B_{Q}^{H}(s)\right\} d v \|_{X}^{2} \\
& -\int_{0}^{T} S(T-s) f(s, x(s-\rho(s))) d s \\
& -\int_{0}^{T} S(T-s) g(s, x(s-\eta(s))) d W(s) \\
& 2 \mathbb{E}\left\|K_{71}(\varepsilon)\right\|_{X}^{2}+2 \mathbb{E}\left\|K_{72}(\varepsilon)\right\|_{X}^{2} . \\
&
\end{aligned}
$$

Applying conditions (C.1)-(C.7) and Lemmas 2-4 to $\mathbb{E}\left\|K_{71}(\varepsilon)\right\|_{X}^{2}$, we get

$$
\begin{aligned}
& \mathbb{E}\left\|K_{71}(\varepsilon)\right\|_{X}^{2} \leq 7 M^{2} M_{B}^{2} M_{\omega}^{2} \mathbb{E}\left\|\int_{t}^{t+\varepsilon} x_{1} d v\right\|_{X}^{2} \\
& +7 M^{2} M_{B}^{2} M_{\omega}^{2} \mathbb{E} \| \int_{t}^{t+\varepsilon} M[x(0)
\end{aligned}
$$

$$
\begin{aligned}
& +h(0, x(-r(0)))] d v \|_{X}^{2} \\
& +7 M^{2} M_{B}^{2} M_{\omega}^{2} \mathbb{E}\left\|\int_{t}^{t+\varepsilon} h(T, x(T-r(T))) d v\right\|_{X}^{2} \\
& +7 M^{2} M_{B}^{2} M_{\omega}^{2} \mathbb{E} \| \int_{t}^{t+\varepsilon} \int_{0}^{T}(-A)^{1-\beta} S(T-s)(-A)^{\beta} \\
& \cdot h(s, x(s-r(s))) d s d v \|_{X}^{2} \\
& +7 M^{2} M_{B}^{2} M_{\omega}^{2} \mathbb{E} \| \int_{t}^{t+\varepsilon} \int_{0}^{T} S(T-s) \\
& \cdot f(s, x(s-\rho(s))) d s d v \|_{X}^{2} \\
& +7 M^{2} M_{B}^{2} M_{\omega}^{2} \mathbb{E} \| \int_{t}^{t+\varepsilon} \int_{0}^{T} S(T-s) \\
& \cdot g(s, x(s-\eta(s))) d W(s) d v \|_{X}^{2} \\
& +7 M^{2} M_{B}^{2} M_{\omega}^{2} \mathbb{E} \| \int_{t}^{t+\varepsilon} \int_{0}^{T} S(T-s) \\
& \cdot \sigma(s) d B_{Q}^{H}(s) d v \|_{X}^{2} \\
& \leq 7 M^{2} M_{B}^{2} M_{\omega}^{2} \varepsilon^{2} \mathbb{E}\left\|x_{1}\right\|_{X}^{2}+7 M^{4} M_{B}^{2} M_{\omega}^{2} \varepsilon^{2} \mathbb{E} \| x(0) \\
& +h(0, x(-r(0)))\left\|_{X}^{2}+7 M^{2} M_{B}^{2} M_{\omega}^{2} C_{h}\right\|(-A)^{-\beta} \|^{2} \\
& \cdot \varepsilon^{2}\left(1+\sup _{s \in[-\tau, T]} \mathbb{E}\|x(s)\|_{X}^{2}\right) \\
& +7 M^{2} M_{B}^{2} M_{\omega}^{2} M_{1-\beta}^{2} C_{h} \beta^{-2} T^{2 \beta} \varepsilon^{2}(1 \\
& \left.+\sup _{s \in[-\tau, T]} \mathbb{E}\|x(s)\|_{X}^{2}\right)+7 M^{4} M_{B}^{2} M_{\omega}^{2} C_{f} T^{2} \varepsilon^{2}(1 \\
& \left.+\sup _{s \in[-\tau, T]} \mathbb{E}\|x(s)\|_{X}^{2}\right)+7 M^{4} M_{B}^{2} M_{\omega}^{2} C_{g} T \varepsilon^{2}(1 \\
& \left.+\sup _{s \in[-\tau, T]} \mathbb{E}\|x(s)\|_{X}^{2}\right)+7 M^{4} M_{B}^{2} M_{\omega}^{2} c_{H} H(2 H-1) \\
& \cdot T^{2 H-1} \mathcal{E}^{2} \int_{0}^{T}\|\sigma(s)\|_{\mathcal{L}_{\mathrm{Q}^{(2)}}}^{2} d s .
\end{aligned}
$$

Then $\mathbb{E}\left\|K_{71}(\varepsilon)\right\|_{X}^{2} \rightarrow 0$ as $\varepsilon \rightarrow 0$. Using the similar technique to $\mathbb{E}\left\|K_{72}(\varepsilon)\right\|_{X}^{2}$, we have

$$
\begin{aligned}
& \mathbb{E}\left\|K_{72}(\varepsilon)\right\|_{X}^{2} \\
& \quad \leq 7 M_{B}^{2} M_{\omega}^{2} \mathbb{E}\left\|\int_{0}^{t}(S(t+\varepsilon-v)-S(t-v)) x_{1} d v\right\|_{X}^{2}
\end{aligned}
$$




$$
\begin{aligned}
& +7 M_{B}^{2} M_{\omega}^{2} \mathbb{E} \| \int_{0}^{t}(S(t+\varepsilon-v)-S(t-v)) \\
& \cdot M[x(0)+h(0, x(-r(0)))] d v \|_{X}^{2} \\
& +7 M_{B}^{2} M_{\omega}^{2} \mathbb{E} \| \int_{0}^{t}(S(t+\varepsilon-v)-S(t-v)) \\
& h(T, x(T-r(T))) d v \|_{X}^{2} \\
& +7 M_{B}^{2} M_{\omega}^{2} \mathbb{E} \| \int_{0}^{t}(S(t+\varepsilon-v)-S(t-v)) \\
& \cdot \int_{0}^{T} A S(T-s) h(s, x(s-r(s))) d s d v \|_{X}^{2} \\
& +7 M_{B}^{2} M_{\omega}^{2} \mathbb{E} \| \int_{0}^{t}(S(t+\varepsilon-v)-S(t-v)) \\
& \cdot \int_{0}^{T} S(T-s) f(s, x(s-\rho(s))) d s d v \|_{X}^{2} \\
& +7 M_{B}^{2} M_{\omega}^{2} \mathbb{E} \| \int_{0}^{t}(S(t+\varepsilon-v)-S(t-v)) \\
& \cdot \int_{0}^{T} S(T-s) g(s, x(s-\eta(s))) d W(s) d v \|_{X}^{2} \\
& +7 M_{B}^{2} M_{\omega}^{2} \mathbb{E} \| \int_{0}^{t}(S(t+\varepsilon-v)-S(t-v)) \\
& \cdot \int_{0}^{T} S(T-s) \sigma(s) d B_{Q}^{H}(s) d v \|_{X}^{2}
\end{aligned}
$$

$$
\begin{aligned}
& \leq\left\{7 M_{B}^{2} M_{\omega}^{2} \mathbb{E}\left\|x_{1}\right\|_{X}^{2}+7 M^{2} M_{B}^{2} M_{\omega}^{2} \mathbb{E} \| x(0)\right. \\
& +h(0, x(-r(0)))\left\|_{X}^{2}+7 M_{B}^{2} M_{\omega}^{2} C_{h}\right\|(-A)^{-\beta} \|^{2}(1 \\
& \left.+\sup _{s \in[-\tau, T]} \mathbb{E}\|x(s)\|_{X}^{2}\right) \\
& +7 M_{B}^{2} M_{\omega}^{2} M_{1-\beta}^{2} C_{h} \beta^{-2} T^{2 \beta}(1 \\
& \left.+\sup _{s \in[-\tau, T]} \mathbb{E}\|x(s)\|_{X}^{2}\right)+7 M^{2} M_{B}^{2} M_{\omega}^{2} C_{f} T^{2}(1 \\
& \left.+\sup _{s \in[-\tau, T]} \mathbb{E}\|x(s)\|_{X}^{2}\right)+7 M^{2} M_{B}^{2} M_{\omega}^{2} C_{g} T(1 \\
& \left.+\sup _{s \in[-\tau, T]} \mathbb{E}\|x(s)\|_{X}^{2}\right)+7 M^{2} M_{B}^{2} M_{\omega}^{2} c_{H} H(2 H-1) \\
& \left.\cdot T^{2 H-1} \int_{0}^{T}\|\sigma(s)\|_{\mathscr{L}^{0}(2)}^{2} d s\right\} \\
& \times\left(\int_{0}^{t}(S(t+\varepsilon-v)-S(t-v)) d v\right)^{2}
\end{aligned}
$$

Then $\mathbb{E}\left\|K_{72}(\varepsilon)\right\|_{X}^{2} \rightarrow 0$ as $\varepsilon \rightarrow 0$.

Thus, $\pi$ is indeed continuous in mean square on $[0, T]$. So we conclude that $\pi\left(S_{T}\right) \subset S_{T}$.

Last, we will show that $\pi$ is a contraction mapping in $S_{T_{1}}$ with some $0<T_{1} \leq T$. Let $x, y \in S_{T}$, as proceeding as we did previously; we can obtain

$$
\begin{aligned}
\mathbb{E} & \|\pi(x)(t)-\pi(y)(t)\|_{X}^{2} \leq \frac{1}{k} \mathbb{E}\|h(t, x(t-r(t)))-h(t, y(t-r(t)))\|_{X}^{2} \\
+ & \frac{7}{1-k}\left\{\mathbb{E}\left\|\int_{0}^{t}(-A)^{1-\beta} S(t-s)(-A)^{\beta}[h(s, x(s-r(s)))-h(s, y(s-r(s)))] d s\right\|_{X}^{2}\right. \\
+ & \mathbb{E}\left\|\int_{0}^{t} S(t-s)[f(s, x(s-\rho(s)))-f(s, y(s-\rho(s)))] d s\right\|_{X}^{2} \\
+ & \mathbb{E}\left\|\int_{0}^{t} S(t-s)[g(s, x(s-\eta(s)))-g(s, y(s-\eta(s)))] d W(s)\right\|_{X}^{2} \\
+ & \mathbb{E}\left\|\int_{0}^{t} S(t-v) B \omega^{-1}[h(T, x(T-r(T)))-h(T, y(T-r(T)))] d v\right\|_{X}^{2} \\
+ & \mathbb{E}\left\|\int_{0}^{t} S(t-v) B \omega^{-1} \int_{0}^{T} A S(T-s)[h(s, x(s-r(s)))-h(s, y(s-r(s)))] d s d v\right\|_{X}^{2} \\
+ & \mathbb{E}\left\|\int_{0}^{t} S(t-v) B \omega^{-1} \int_{0}^{T} S(T-s)[f(s, x(s-\rho(s)))-f(s, y(s-\rho(s)))] d s d v\right\|_{X}^{2} \\
+ & \left.\mathbb{E}\left\|\int_{0}^{t} S(t-v) B \omega^{-1} \int_{0}^{T} S(T-s)[g(s, x(s-\eta(s)))-g(s, y(s-\eta(s)))] d W(s) d v\right\|_{X}^{2}\right\} .
\end{aligned}
$$


Thus

$$
\begin{aligned}
& \mathbb{E}\|\pi(x)(t)-\pi(y)(t)\|^{2} \\
& \leq k \mathbb{E}\|x(t-r(t))-y(t-r(t))\|_{X}^{2}+\frac{7 M_{1-\beta}^{2} L_{h}^{2} t^{2 \beta}}{(1-k) \beta^{2}} \\
& \sup _{s \in[-\tau, T]} \mathbb{E}\|x(s)-y(s)\|_{X}^{2}+\frac{7 M^{2} L_{f}^{2} t^{2}}{1-k} \\
& \sup _{s \in[-\tau, T]} \mathbb{E}\|x(s)-y(s)\|_{X}^{2}+\frac{7 M^{2} L_{g}^{2} t}{1-k} \\
& \text {. } \sup _{s \in[-\tau, T]} \mathbb{E}\|x(s)-y(s)\|_{X}^{2} \\
& +\frac{7 M^{2} M_{B}^{2} M_{\omega}^{2} L_{h}^{2}\left\|(-A)^{-\beta}\right\|^{2} t^{2}}{1-k} \\
& \text { - } \mathbb{E}\|x(T-r(T))-y(T-r(T))\|_{X}^{2} \\
& +\frac{7 M^{2} M_{1-\beta}^{2} M_{B}^{2} M_{\omega}^{2} L_{h}^{2} T^{2 \beta} t^{2}}{(1-k) \beta^{2}} \\
& \cdot \sup _{s \in[-\tau, T]} \mathbb{E}\|x(s)-y(s)\|_{X}^{2}+\frac{7 M^{4} M_{B}^{2} M_{\omega}^{2} L_{f}^{2} T^{2} t^{2}}{1-k} \\
& \sup _{s \in[-\tau, T]} \mathbb{E}\|x(s)-y(s)\|_{X}^{2}+\frac{7 M^{4} M_{B}^{2} M_{\omega}^{2} L_{g}^{2} T t^{2}}{1-k} \\
& \cdot \sup _{s \in[-\tau, T]} \mathbb{E}\|x(s)-y(s)\|_{X}^{2} .
\end{aligned}
$$

Hence

$$
\begin{aligned}
& \sup _{t \in[-\tau, T]} \mathbb{E}\|\pi(x)(t)-\pi(y)(t)\|_{X}^{2} \\
& \quad \leq b(t) \sup _{t \in[-\tau, T]} \mathbb{E}\|x(t)-y(t)\|_{X}^{2},
\end{aligned}
$$

where

$$
\begin{aligned}
b(t)= & k+\frac{7 M_{1-\beta}^{2} L_{h}^{2} t^{2 \beta}}{(1-k) \beta^{2}}+\frac{7 M^{2} L_{f}^{2} t^{2}}{1-k}+\frac{7 M^{2} L_{g}^{2} t}{1-k} \\
& +\frac{7 M^{2} M_{B}^{2} M_{\omega}^{2} L_{h}^{2}\left\|(-A)^{-\beta}\right\|^{2} t^{2}}{1-k} \\
& +\frac{7 M^{2} M_{1-\beta}^{2} M_{B}^{2} M_{\omega}^{2} L_{h}^{2} T^{2 \beta} t^{2}}{(1-k) \beta^{2}} \\
& +\frac{7 M^{4} M_{B}^{2} M_{\omega}^{2} L_{f}^{2} T^{2} t^{2}}{1-k}+\frac{7 M^{4} M_{B}^{2} M_{\omega}^{2} L_{g}^{2} T t^{2}}{1-k} .
\end{aligned}
$$

By condition (C.3), we know that $b(0)=k<1$ then there exists $0<T_{1} \leq T$ such that $0<\gamma\left(T_{1}\right)<1$ which shows that $\pi$ is a contraction mapping in $S_{T_{1}}$. Then $\pi$ has a unique fixed point in $S_{T_{1}}$, which is a unique mild solution of (1) on $\left[-\tau, T_{1}\right]$. Repeating this procedure can be used to extend the mild solution to the entire interval $[-\tau, T]$. Obviously, $\pi(x)(T)=x_{1}$ which means that system (1) is controllable on $[-\tau, T]$. This completes the proof.

\section{An Example}

In this section, we provide an example to illustrate our main results. Consider the following NSDPDE with finite delays $\tau_{1}$, $\tau_{2}$, and $\tau_{3}\left(0 \leq \tau_{i} \leq \tau<\infty, i=1,2,3\right)$ :

$$
\begin{aligned}
& d\left[x(t, \xi)-H\left(t, x\left(t-\tau_{1}, \xi\right)\right)\right] \\
& =\left[\frac{\partial^{2}}{\partial \xi^{2}} x(t, \xi)+\mu(t, \xi)+F\left(t, x\left(t-\tau_{2}, \xi\right)\right)\right] d t \\
& \quad+G\left(t, x\left(t-\tau_{3}, \xi\right)\right) d W(t)+\sigma(t) d B_{Q}^{H}(t), \\
& 0 \leq \xi \leq \pi, \quad t \in[0, T], \\
& x(t, 0)=x(t, \pi)=0, \quad t \in[0, T], \\
& x(t, \xi)=\varphi(t, \xi), \quad t \in[-\tau, 0], 0 \leq \xi \leq \pi,
\end{aligned}
$$

where $W(t)$ is a cylindrical Brownian motion and $B_{\mathrm{Q}}^{H}(t)$ is a cylindrical fractional Brownian motion in $X$.

Let $X=L^{2}([0, \pi])$ and define the operator $A: D(A) \subset$ $X \rightarrow X$ by $A=\partial^{2} / \partial \xi^{2}$ with the domain

$$
\begin{aligned}
& D(A):=\{z \\
& \quad \in X, z, z^{\prime} \text { are absolutely continuous on, } z^{\prime \prime} \\
& \quad \in X, z(0)=z(\pi)=0\} .
\end{aligned}
$$

It is well known that an analytic semigroup $\{S(t)\}_{t \geq 0}$ is generated by the operator $A$ in $X$ satisfying $\|S(t)\|_{X} \leq e^{-t}$. Furthermore,

$$
A u=\sum_{i=1}^{\infty} n^{2}\left\langle u, e_{n}\right\rangle e_{n}, \quad u \in D(A),
$$

where $e_{n}(x)=\sqrt{2 / \pi} \sin (n x)$ is a complete orthonormal set of eigenvectors of $A$. Then, the following properties hold:

(i) If $y \in D(A)$, then $A y=\sum_{n=1}^{\infty} n^{2}\left\langle y, e_{n}\right\rangle e_{n}$.

(ii) For each $y \in X, A^{-1 / 2} y=\sum_{n=1}^{\infty}(1 / n)\left\langle y, e_{n}\right\rangle e_{n}$. In particular, $\left\|A^{-1 / 2}\right\|_{X}=1$.

(iii) The operator $A^{1 / 2}$ is given by

$$
A^{1 / 2} y=\sum_{n=1}^{\infty} n\left\langle y, x_{n}\right\rangle e_{n}
$$

on the space $D\left[A^{1 / 2}\right]=\left\{y(\cdot) \in X, \sum_{n=1}^{\infty} n\left\langle y, e_{n}\right\rangle e_{n} \Sigma\right\}$.

We assume that the following conditions hold:

(1) Let $B: U \rightarrow X$ be a bounded linear operator defined by

$$
B u(t)(\xi)=\mu(t, \xi), \quad 0 \leq \xi \leq \pi, u \in L^{2}([0, \pi], U) .
$$


(2) The operator $\omega: \in L^{2}([0, \pi], U) \rightarrow X$ is given by $\omega u(\xi)=\int_{0}^{T} S(T-s) \mu(s, \xi) d s, \quad 0 \leq \xi \leq \pi$,

has a bounded invertible operator $\omega^{-1}$, and satisfies condition (C.7). For the construction of the operator $\omega$ and its inverse, see [26] for more details.

(3) There exist nonnegative constants $L_{h}<1, L_{f}$, and $L_{g}$ such that, for all $t \in[-\tau, T], x, y \in X$, and $\xi \in[0, \pi]$,

$$
\begin{aligned}
& \|F(t, x(t, \xi))-F(t, y(t, \xi))\|_{X} \\
& \quad \leq L_{f}\|x(t, \xi)-y(t, \xi)\|_{X}, \\
& \|G(t, x(t, \xi))-G(t, y(t, \xi))\|_{X} \\
& \quad \leq L_{g}\|x(t, \xi)-y(t, \xi)\|_{X}, \\
& \left\|(-A)^{1 / 2} H(t, x(t, \xi))-(-A)^{1 / 2} H(t, y(t, \xi))\right\|_{X} \\
& \quad \leq L_{h}\|x(t, \xi)-y(t, \xi)\|_{X} .
\end{aligned}
$$

(4) There exist nonnegative constants $C_{f}, C_{g}$, and $C_{h}$, such that, for all $t \in[-\tau, T], x \in X$, and $\xi \in[0, \pi]$,

$$
\begin{array}{r}
\|F(t, x(t, \xi))\|_{X}^{2} \leq C_{f}\left(1+\|x(t, \xi)\|_{X}^{2}\right), \\
\|G(t, x(t, \xi))\|_{X}^{2} \leq C_{g}\left(1+\|x(t, \xi)\|_{X}^{2}\right), \\
\left\|(-A)^{1 / 2} H(t, x(t, \xi))\right\|_{X}^{2} \leq C_{h}\left(1+\|x(t, \xi)\|_{X}^{2}\right) .
\end{array}
$$

(5) The mapping $(-A)^{1 / 2} H$ is continuous in mean square on $[-\tau, T]$ : for all $t \in[-\tau, T], x \in X$, and $\xi \in[0, \pi]$, we have

$$
\begin{aligned}
\lim _{t \rightarrow s} \mathbb{E} & \|(-A)^{1 / 2} H(t, x(t, \xi)) \\
- & (-A)^{1 / 2} H(s, x(s, \xi)) \|_{X}^{2}=0 .
\end{aligned}
$$

(6) The mapping $\sigma:[0, T] \rightarrow \mathscr{L}_{\mathrm{Q}^{(2)}}^{0}\left(Y_{2} ; X\right)$ satisfies

$$
\int_{0}^{T}\|\sigma(s)\|_{\mathscr{L}_{Q^{(2)}}^{0}}^{2} d s<\infty, \quad \forall T>0 .
$$

Define the operators $F, G$, and $H: \mathbb{R}^{+} \times L^{2}([0, \pi]) \rightarrow$ $L^{2}([0, \pi])$ by

$$
\begin{aligned}
& h(t, \varphi)(\xi)=H\left(t, \varphi\left(-\tau_{1}\right)(\xi)\right) \\
& \qquad \text { for } \xi \in[0, \pi], \varphi \in L^{2}([0, \pi]), \\
& f(t, \varphi)(\xi)=F\left(t, \varphi\left(-\tau_{2}\right)(\xi)\right) \\
& \quad \text { for } \xi \in[0, \pi], \varphi \in L^{2}([0, \pi]), \\
& g(t, \varphi)(\xi)=G\left(t, \varphi\left(-\tau_{3}\right)(\xi)\right)
\end{aligned}
$$$$
\text { for } \xi \in[0, \pi], \varphi \in L^{2}([0, \pi]) \text {. }
$$

If we put

$$
\begin{aligned}
x(t)(\xi) & =x(t, \xi), \quad t \in[0, T], \xi \in[0, \pi], \\
x(t, \xi) & =\varphi(t, \xi), \quad t \in[-\tau, 0], \xi \in[0, \pi],
\end{aligned}
$$

then problem (41) can be written in the abstract form

$$
\begin{aligned}
& d[x(t)+h(t, x(t-r(t)))] \\
& =[A x(t)+f(t, x(t-\rho(t)))+B u(t)] d t \\
& \quad+g(t, x(t-\eta(t))) d W(t)+\sigma(t) d B_{\mathrm{Q}}^{H}(t), \\
& \quad 0 \leq t \leq T, \\
& x(s)=\varphi(s), \quad-\tau \leq s \leq 0 .
\end{aligned}
$$

Thus all the conditions of Theorems 6 and 9 are fulfilled. Therefore, we conclude that the system (41) has a unique mild solution which is controllable on $[-\tau, T]$.

\section{Conclusion}

In this paper, by using the Banach fixed point theorem, we obtain some sufficient conditions ensuring the existence, uniqueness, and controllability of neutral stochastic delay partial differential equations driven by standard Brownian motion and fractional Brownian motion. In our next paper, we will explore the existence, uniqueness, and controllability problems with non-Lipschitz condition.

\section{Conflicts of Interest}

The authors declare that there are no conflicts of interest regarding the publication of this paper.

\section{Acknowledgments}

This research was supported by the National Natural Science Foundation of China under Grant no. 11271093 and the Innovation Research for the Postgraduates of Guangzhou University under Grant no. 2017GDJC-D09.

\section{References}

[1] E. Alos, O. Mazet, and D. Nualart, "Stochastic calculus with respect to Gaussian processes," Annals of Probability, vol. 29, no. 2, pp. 766-801, 2001.

[2] T. Caraballo, M. J. Garrido-Atienza, and T. Taniguchi, "The existence and exponential behavior of solutions to stochastic delay evolution equations with a fractional Brownian motion," Nonlinear Analysis. Theory, Methods \& Applications, vol. 74, no. 11, pp. 3671-3684, 2011.

[3] B. Maslowski and D. Nualart, "Evolution equations driven by a fractional Brownian motion," Journal of Functional Analysis, vol. 202, no. 1, pp. 277-305, 2003.

[4] D. Nualart, Fractional Brownian Motion: Stochastic Calculus and Applications, vol. 3, Eur. Math. Soc, Zürich, 2006.

[5] D. Nualart, The Malliavin Calculus and Related Topics, Springer, Berlin, 2006. 
[6] H. M. Ahmed, "Approximate controllability of impulsive neutral stochastic differential equations with fractional Brownian motion in a Hilbert space," Advances in Difference Equations, vol. 113, 11 pages, 2014.

[7] P. Balasubramaniam and S. K. Ntouyas, "Controllability for neutral stochastic functional differential inclusions with infinite delay in abstract space," Journal of Mathematical Analysis and Applications, vol. 324, no. 1, pp. 161-176, 2006.

[8] B. Boufoussi and S. Hajji, "Neutral stochastic functional differential equations driven by a fractional Brownian motion in a Hilbert space," Statistics \& Probability Letters, vol. 82, no. 8, pp. 1549-1558, 2012.

[9] J. Cui and L. Yan, "Controllability of neutral stochastic evolution equations driven by fractional Brownian motion," Acta Mathematica Scientia B, vol. 37, no. 1, pp. 108-118, 2017.

[10] S. Hajji and E. Lakhel, "Existence and uniqueness of mild solutions to neutral SFDE driven by a fractional Brownian motion with non-Lipschitz coefficients," Journal of Numerical Mathematics and Stochastics, vol. 7, no. 1, pp. 14-29, 2015.

[11] E. H. Lakhel, "Controllability of neutral stochastic functional integro-differential equations driven by fractional Brownian motion," Stochastic Analysis and Applications, vol. 34, no. 3, pp. 427-440, 2016.

[12] M. Li and M. Liu, "Approximate controllability of semilinear neutral stochastic integrodifferential inclusions with infinite delay," Discrete Dynamics in Nature and Society, Article ID 420826, Art. ID 420826, 16 pages, 2015.

[13] W. Liu and J. Luo, "Neutral stochastic differential equations driven by Brownian motion and fractional BROwnian motion in a Hilbert space," Publicationes Mathematicae, vol. 87, no. 1-2, pp. 235-253, 2015.

[14] J. Luo, "Fixed points and stability of neutral stochastic delay differential equations," Journal of Mathematical Analysis and Applications, vol. 334, no. 1, pp. 431-440, 2007.

[15] J. Luo, "Exponential stability for stochastic neutral partial functional differential equations," Journal of Mathematical Analysis and Applications, vol. 355, no. 1, pp. 414-425, 2009.

[16] J. Y. Park, P. Balasubramaniam, and N. Kumaresan, "Controllability for neutral stochastic functional integrodifferential infinite delay systems in abstract space," Numerical Functional Analysis and Optimization, vol. 28, no. 11-12, pp. 1369-1386, 2007.

[17] P. Cheridito, "Mixed fractional Brownian motion," Bernoulli Society for Mathematical Statistics and Probability, vol. 7, no. 6, pp. 913-934, 2001.

[18] Y. S. Mishura and S. V. Posashkova, "Stochastic differential equations driven by a Wiener process and fractional Brownian motion: convergence in BESov space with respect to a parameter," Computers \& Mathematics with Applications, vol. 62, no. 3, pp. 1166-1180, 2011.

[19] Y. Mishura and G. Shevchenko, "Mixed stochastic differential equations with long-range dependence: Existence, uniqueness and convergence of solutions," Computers \& Mathematics with Applications, vol. 64, no. 10, pp. 3217-3227, 2012.

[20] G. Shevchenko and T. Shalaiko, "Malliavin regularity of solutions to mixed stochastic differential equations," Statistics \& Probability Letters, vol. 83, no. 12, pp. 2638-2646, 2013.

[21] G. Shevchenko, "Mixed fractional stochastic differential equations with jumps," Stochastics. An International Journal of Probability and Stochastic Processes, vol. 86, no. 2, pp. 203-217, 2014.
[22] J. Klamka, "Stochastic controllability of linear systems with delay in control," Bulletin of the Polish Academy of Sciences Technical Sciences, vol. 55, pp. 23-29, 2007.

[23] S. Tindel, C. A. Tudor, and F. Viens, "Stochastic evolution equations with fractional Brownian motion," Probability Theory and Related Fields, vol. 127, no. 2, pp. 186-204, 2003.

[24] G. Da Prato and J. Zabczyk, Stochastic Equations in Infinite Dimensions, vol. 44, Cambridge University Press, Cambridge, UK, 1992.

[25] A. Pazy, Semigruops of Linear Operators and Applications to Partial Differential Equations, vol. 44 of Applied Mathematical Sciences, Springer, 1983.

[26] M. D. Quinn and N. Carmichael, "An approach to nonlinear control problems using fixed-point methods, degree theory and pseudo-inverses," Numerical Functional Analysis and Optimization, vol. 7, no. 2-3, pp. 197-219, 1984/85. 


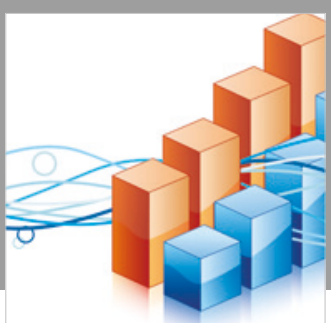

Advances in

Operations Research

\section{-n-m}
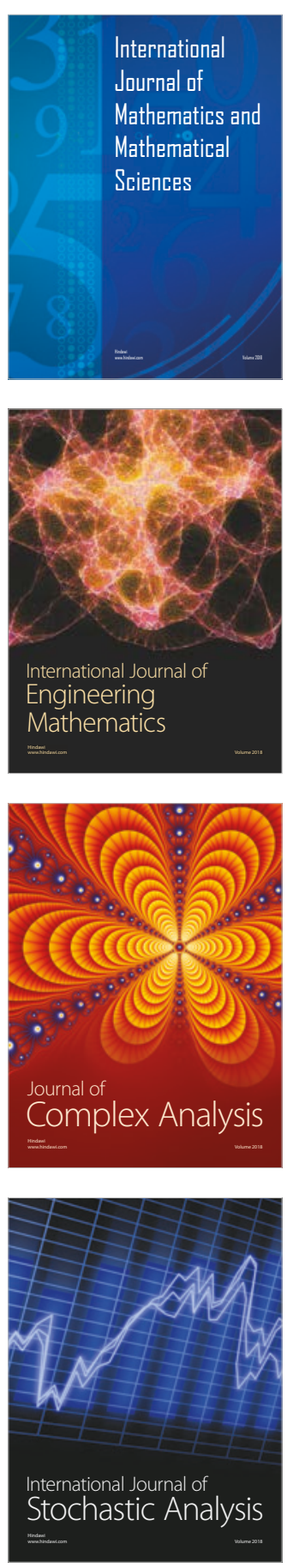
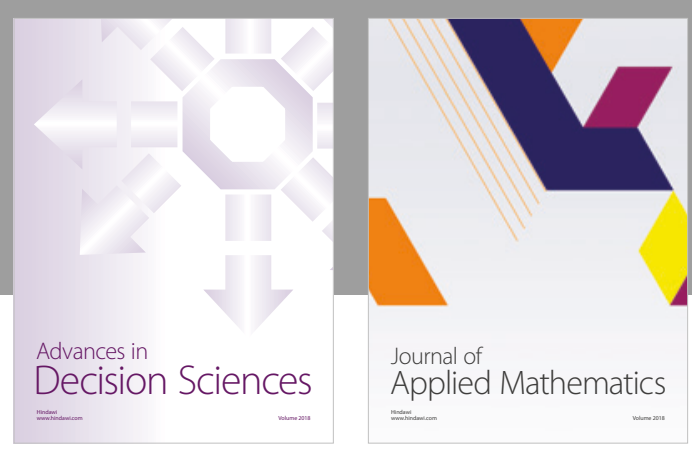

Journal of

Applied Mathematics
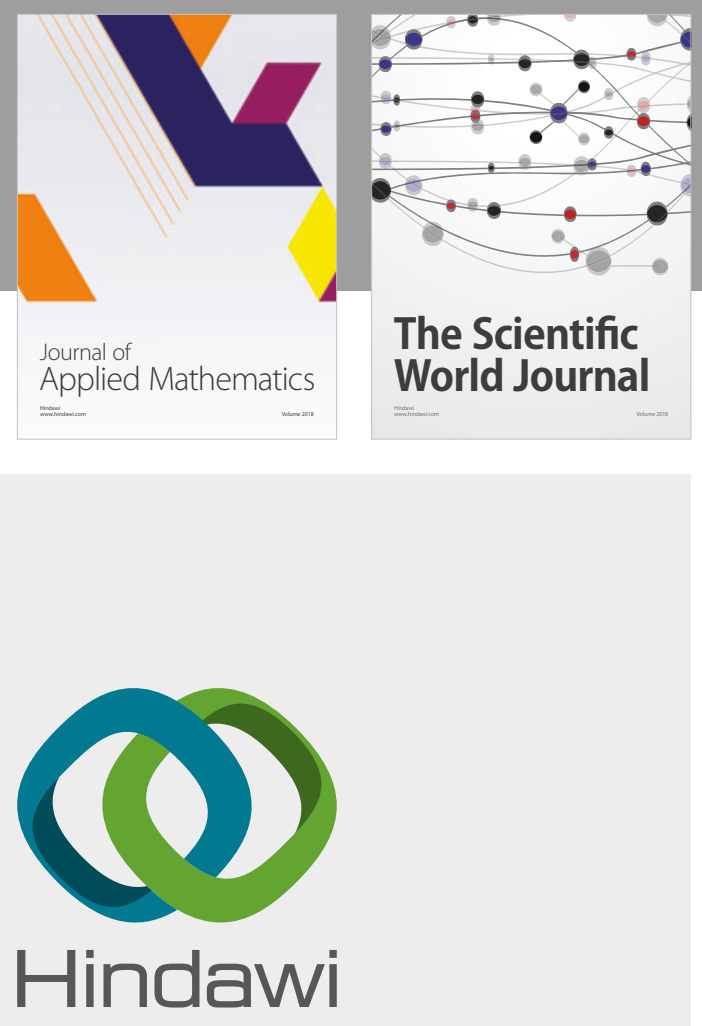

Submit your manuscripts at

www.hindawi.com

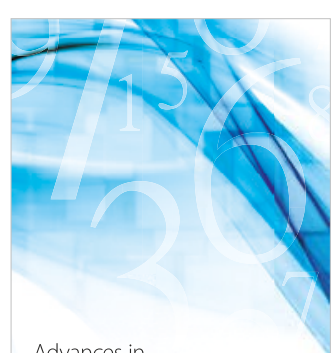

Advances in
Numerical Analysis
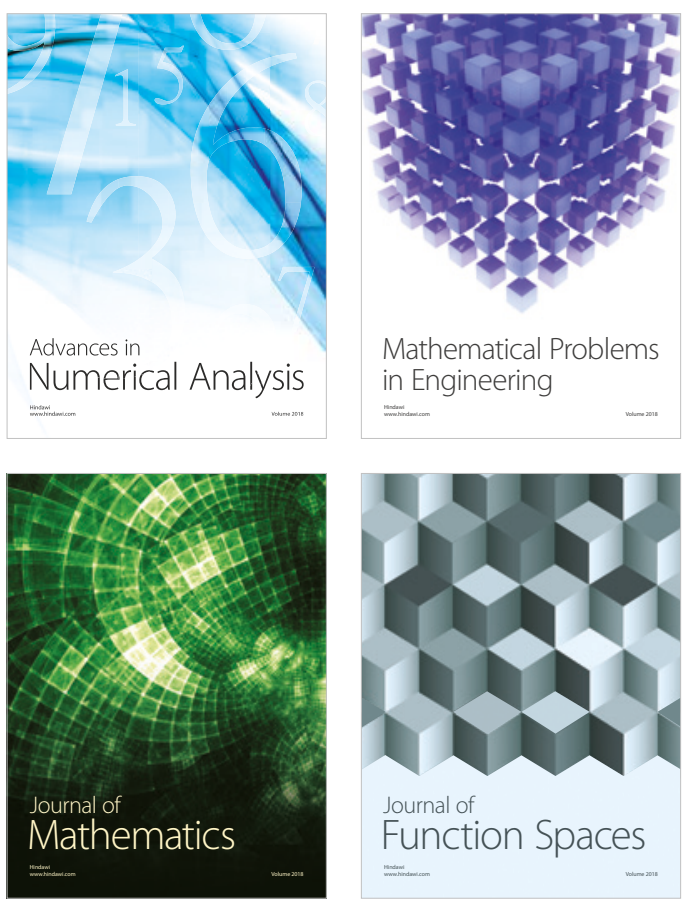

Mathematical Problems in Engineering

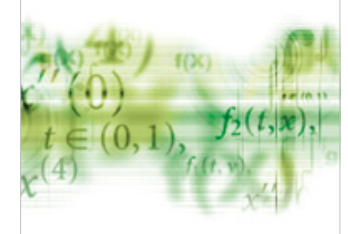

International Journal of

Differential Equations

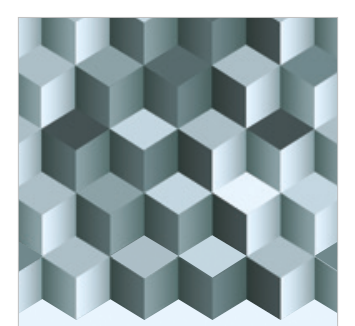

Journal of

Function Spaces

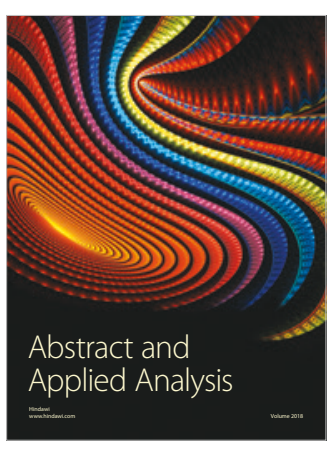

The Scientific

World Journal

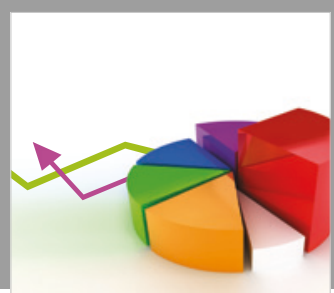

Journal of

Probability and Statistics
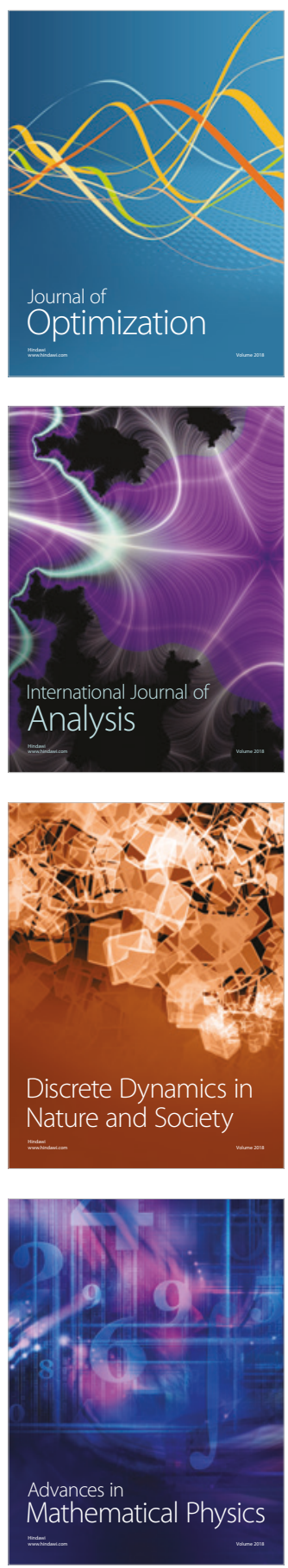